\title{
CARACTERIZACIÓN DE Pseudotsuga menziesii (MIRB.) FRANCO EN EL CENTRO DE MÉXICO. IMPLICACIONES PARA SU CONSERVACIÓN
}

\section{CHARACTERIZATION OF POPULATIONS OF Pseudotsuga menziesii (MIRB.) FRANCO IN CENTRAL MÉXICO. IMPLICATIONS FOR CONSERVATION}

\author{
Araceli Ventura Ríos ${ }^{1}$, Javier López Upton ${ }^{1 *}$, J. Jesús Vargas Hernández ${ }^{1}$ \\ y Vidal Guerra de la $\mathrm{Cruz}^{2}$
}

\begin{abstract}
${ }^{1}$ Postgrado Forestal, Colegio de Postgraduados, Campus Montecillo. Km 36.5 Carretera México-Texcoco. 56230, Montecillo, Texcoco, Estado de México Tel y Fax 01 (595) 95202 56. ${ }^{2}$ Instituto Nacional de Investigaciones Forestales Agrícolas y Pecuarias, Tlaxcala. Km. 2.5 Carr. Tlaxcala-Santa Ana Chiautempan. 90800, Tlaxcala, Tlax.
\end{abstract}

* Autor para correspondencia (uptonj@colpos.mx)

\section{RESUMEN}

El objetivo de este estudio fue describir las condiciones ecológicas de 29 poblaciones de Pseudotsuga menziesii (Mirb.) Franco en los Estados de Hidalgo, Puebla y Tlaxcala, ubicados en la región central de México. Se estimó una población total de 9029 árboles adultos en 682 ha que se ubican desde 2450 a $3400 \mathrm{~m}$, en suelos con pH ligeramente ácidos, ricos en materia orgánica y clase textural francoarenosa y franco. Las localidades se ubican en clima templado, con temperatura media que oscila de 10.1 a $14.0{ }^{\circ} \mathrm{C}$, y una precipitación media anual de 611 a $1109 \mathrm{~mm}$. P. menziesii convive con Abies religiosa (Kunth) Schltdl. et Cham. y varias especies de los géneros Pinus y Quercus, en exposiciones N, NO y NE. El tamaño poblacional varía de 4 a 1450 árboles maduros; 11 de las poblaciones presentan menos de 100 árboles adultos. La regeneración es escasa en todas ellas y existe gran fragmentación en su distribución natural. Con base en un análisis de agrupamiento se detectó similitud entre las poblaciones de Hidalgo (Zona I) y entre las poblaciones de Tlaxcala y Puebla (Zona II), cada zona con seis grupos de poblaciones. Se recomienda intercambiar material genético dentro de grupos y ocasionalmente entre grupos, para restablecer el flujo génico entre poblaciones y propiciar mayor variabilidad genética. Se discuten algunos aspectos clave para la conservación de estas pequeñas poblaciones.

Palabras clave: Abeto Douglas, agrupamiento de poblaciones, caracterización ecológica, conservación

\section{SUMMARY}

The objective of this study was to describe the ecological conditions of 29 stands of Pseudotsuga menziesii (Mirb.) Franco located in the states of Hidalgo, Puebla and Tlaxcala, in Central México. An estimated of 9029 adult Douglas-fir trees are growing in an area of $682 \mathrm{ha}$, located in altitudes between 2450 and $3400 \mathrm{~m}$ on soils with slightly acidic $\mathrm{pH}$, rich in organic matter and sandy loam and loam texture in most cases. The locations have a temperate climate, with an average temperatures ranging from 10.1 to $14.0{ }^{\circ} \mathrm{C}$ and annual rainfall range from 611 to $1109 \mathrm{~mm}$. P. menziesii coexists with Abies eligiosa (Kunth) Schltdl. et Cham. and with several species of the genus Pinus and Quercus, facing N, NW and
NE. Tree population size varies from 4 to 1450 matures trees; 11 stands have less than 100 adult trees. The natural regeneration is low in all of them, and the stands are fragmented within their natural range. Based on a cluster analysis, two clusters of stands were found: one located at Hidalgo State (Zone I), and another grouping in the Tlaxcala and Puebla (Zone II) stands. Each zone has six groups of populations. Genetic material exchange is recommended inside groups and occasionally among groups of populations to restore gene flow and encourage genetic variability. Key aspects are discussed for the conservation of these small stands.

Index words: Douglas-fir, clustering of stands, ecological characterization, conservation.

\section{INTRODUCCIÓN}

Pseudotsuga menziesii (Mirb.) Franco aunque abundante e importante en el oeste de EE.UU. y Canadá (Hermann y Lavender, 1999), en México es escaso y se ha incluido como especie bajo protección especial en la NOM-059-SEMARNAT-2001 (SEMARNAT, 2002a), lo que prohibe su aprovechamiento comercial. A pesar de que la Norma reconoce cuatro especies, estudios recientes indican que todas son P. menziesii (Reyes et al., 2005; Reyes et al., 2006). En México, los árboles de Pseudotsuga o pinabete (abeto Douglas) se encuentran en poblaciones aisladas, frecuentemente mezclados y dominados por otras especies (Domínguez et al., 2004). El pinabete crece en forma natural principalmente en la porción norte de la Sierra Madre Occidental, en los Estados de Chihuahua, Durango, Sonora y Zacatecas, y en la parte norte de la Sierra Madre Oriental en Nuevo León, Coahuila y Tamaulipas. Además, se encuentra en pequeños rodales en la parte oriental del Eje Neovolcánico, en el centro del país (Domínguez, 1994). En el Estado de Oaxaca se localizan dos poblaciones (Debreczy y Rácz, 1995; Del Castillo et al., 2004). 
Aunque no hay un recuento preciso de las poblaciones naturales de $P$. menziesii en el centro de México, se han reportado alteraciones en ellas por el cambio de uso de suelo, el sobrepastoreo, la tala clandestina, la colecta inapropiada de semilla y las plagas, mismas que reducen la cantidad de conos y semillas, y amenazan la permanencia de esta conífera en la región (Mápula et al., 2007; Velasco et al., 2007). Los árboles de $P$. menziesii producen madera de buena calidad (Sanhueza et al., 1998) y son demandados en la época navideña como plantas de ornato, debido a la configuración de la copa y la permanencia de sus hojas una vez cortado el árbol (Álvarez et al., 2007). En el 2007 se importaron 1200000 árboles de navidad, la mayoría de $P$. menziesii provenientes de EE. UU. y Canadá (CONAFOR, 2007). Por su importancia comercial se colecta semilla de manera indiscriminada, que ocasiona daños a los árboles, además de que tienen problemas para producir semilla y generar nuevos individuos (Mápula $e t$ al., 2007; Velasco et al., 2007). Las poblaciones del centro tienen poca variabilidad genética debido a los efectos de depresión endogámica, por lo que se ha sugerido propiciar el intercambio genético (por ejemplo, mediante movimiento de polen) entre las poblaciones del centro (Cruz et al., 2008). La migración asistida de individuos de otras poblaciones mediante plantaciones puede ser útil para aumentar la diversidad genética. La polinización cruzada debería ser entre poblaciones ubicadas en ambientes similares, ya que se han detectado diferencias importantes en las características adaptativas entre poblaciones de Pseudotsuga de México (Acevedo et al., 2006; Reyes et al., 2005; Reyes et al., 2006).

El objetivo de este estudio fue determinar la distribución precisa de $P$. menziesii en el centro de México, conocer el tamaño de las poblaciones, las condiciones ambientales en que se desarrollan y la similitud entre éstas, que permitan establecer actividades de conservación en su hábitat natural.

\section{MATERIALES Y MÉTODOS}

\section{Ubicación y delimitación de las poblaciones de Pseudotsuga menziesii}

El trabajo se realizó en el área de distribución natural de $P$. menziesii en la zona centro de México, en los Estados de Hidalgo, Tlaxcala y Puebla, que se localiza entre $19^{\circ} 10^{\prime}$ y $20^{\circ} 12^{\prime} \mathrm{LN}$ y entre $97^{\circ} 20^{\prime}$ y $98^{\circ} 47^{\prime}$ LO. Para la determinación de la distribución se usó información bibliográfica y la consulta en herbarios, a profesionales y técnicos forestales, así como a propietarios. Se corroboró la ubicación y delimitación de las poblaciones de esta conífera mediante recorridos de campo. Las coordenadas geográficas de los vértices del polígono de cada población se registraron con un GPS (Sistema de Posicionamiento Global) en la proyección NAD27, en el sistema de referencia WGS 84. Los polígonos se transformaron a formato digital y se obtuvo un mapa de distribución de las poblaciones mediante el Sistema de Información Geográfica (SIG) ArcMap 8x${ }^{\circledR}$. En todas las localidades se determinó la altitud con un altímetro, y la exposición del terreno y el porcentaje de pendiente con una brújula azimutal.

\section{Caracterización ecológica de las poblaciones de Pseudotsuga menziesii}

En cada rodal se colectaron tres muestras de suelo de una profundidad de $15-30 \mathrm{~cm}$ (1 $\mathrm{kg}$ de suelo por muestra), en lo que se determinó el pH en solución acuosa (1:2), la textura mediante el método de Bouyucos, densidad aparente (Dap), materia orgánica (MO), el color en húmedo y en seco mediante la escala de colores de Munsell (1990). Los análisis de suelo se hicieron en el Laboratorio de Génesis del Colegio de Postgraduados, basados en la NOM-021-RECNAT-2000 (SEMARNAT, 2002b) y el manual para análisis de suelos de van Reeuwijk (1999). El tipo de clima se determinó con cartas climáticas 1:250 000 (INEGI, 1988). En cada población se obtuvo la temperatura y la precipitación media anual, y un índice de aridez (el cociente de dividir la sumatoria a través del año de la temperatura que ocurre diariamente arriba de $5{ }^{\circ} \mathrm{C}$ entre la precipitación total) (Rehfeldt, 2006; Sáenz et al., 2006), a partir de un modelo climático basado en superficies de interpolación conocidas como "thin plate splines", desarrolladas previamente con el programa ANUSPLIN ${ }^{\circledR}$ versión 4.3 (Hutchinson, 2004).

En cada población se hicieron tres sitios circulares de muestreo de $1000 \mathrm{~m}^{2}$ por el método de muestreo simple aleatorio para determinar el número de individuos adultos de $P$. menziesii. Los árboles que presentaron conos o indicios de eventos reproductivos anteriores, independientemente de sus dimensiones, se consideraron como adultos. Con base en la densidad media de los árboles adultos de $P$. menziesii y con la superficie estimada mediante el Sistema de Información Geográfica, se estimó el tamaño poblacional. En las poblaciones pequeñas se hizo un censo. Además, se registró de manera visual la regeneración de $P$. menziesii y se contaron los individuos de las especies arbóreas asociadas. 


\section{Agrupación de las poblaciones}

Para detectar similitudes ambientales entre poblaciones se utilizaron sólo las variables que pudieran estar asociadas a la capacidad adaptativa de cada población. En el análisis se incluyeron las variables de ubicación geográfica (latitud y longitud) para evitar proponer movimientos de germoplasma muy lejanos (propuesta conservadora). En total, para cada población se obtuvieron valores de 14 variables geográficas y biofísicas: latitud, longitud, altitud, temperatura media anual, precipitación media anual, tipo de clima, índice de aridez, principal especie o género arbóreo asociado, tipo de suelo, $\mathrm{pH}$, materia orgánica, densidad aparente, color y textura del suelo. Se incluyó la principal especie asociada por considerar que ésta pudiera ser indicadora más precisa de ambientes diferentes. Primero se efectuó un análisis de correlación lineal simple entre estas variables, y las que tuvieron correlaciones altas y significativas con otras $(<-$ 0.75 ó > 0.75), se excluyeron con la finalidad de eliminar efectos de colinealidad. La medida en que dos o más variables estén altamente correlacionadas aumenta la ponderación a ellas mismas y tienden a dominar en el análisis de agrupamiento, lo que debe evitarse (Sneath y Sokal, 1973). Con el fin de determinar las poblaciones que poseen similitudes ambientales, se hizo un análisis de agrupamiento con siete variables geográficas y biofísicas: latitud, longitud, tipo de clima, índice de aridez, tipo de vegetación, $\mathrm{pH}$ y textura de suelo. Se utilizó el procedimiento CLUSTER (SAS Institute, 1998) con estandarización por media cero, desviación estándar igual a uno, y el método de ligamiento promedio (opción STD y método AVERAGE), y se graficó el agrupamiento con el procedimiento TREE.

\section{RESULTADOS}

\section{Ubicación y delimitación de las poblaciones de Pseudotsuga menziesii}

Se encontró un total de 29 poblaciones de pinabete distribuidas de manera irregular y dispersas en 12 municipios: Mineral del Chico, Omitlán de Juárez, Mineral del Monte, Pachuca y Singuilucan en el Estado de Hidalgo; Tlaxco, Emiliano Zapata, Terrenate y Altzayanca en Tlaxcala, e Ixtacamaxtitlán, Lafragua y Tlachichuca en Puebla (Cuadro 1). Las poblaciones se ubican entre $19^{\circ} 10^{\prime} 10^{\prime \prime}$ y $20^{\circ} 12^{\prime} 45^{\prime \prime} \mathrm{LN}$ y entre $97^{\circ}$ $18^{\prime} 00^{\prime}$ y $98^{\circ} 47^{\prime} 49$ ” LO, con un intervalo altitudinal de 2450 a $3400 \mathrm{~m}$, con un promedio de $2800 \mathrm{~m}$. La población de Pseudotsuga localizada a una mayor altitud fue en Plan del Baile y la de menor elevación en Vicente Guerrero. La población localizada más al norte es Capula en Mineral del Chico, y la más austral es Plan del Baile en Tlachichuca. Esta última se localiza más al este y San José Capulines se localiza más al oeste (seguida por Capula). El área total que cubren las 29 poblaciones es de aproximadamente 682 ha; la de mayor extensión es Villarreal con 78 ha (11.4\% del total), en tanto que la menor es Morán con 0.5 ha. Las poblaciones de Pseudotsuga del centro de México tienen una extensión reducida y están fragmentadas e incluso aisladas entre sí (Figura 1). No se tiene información de la extensión de $P$. menziesii en el norte de México; EE. UU. y Canadá reportan 14 millones y 4.5 millones de ha, respectivamente (Herman y Lavender, 1999).

La altitud de las poblaciones estudiadas de $P$. menziesii oscila de 2450 a $3400 \mathrm{~m}$ (Cuadro 2), la mayoría alrededor de $2900 \mathrm{~m}$. La pendiente de los terrenos donde se desarrolla esta especie es mayor a $50 \%$. Es común ubicar a la especie en barrancas pronunciadas y lomeríos. Estos sitios son favorecidos por condiciones de sombra y humedad, y generalmente no son propicios para el desmonte con fines agrícolas o pecuarios. El tipo de exposición predominante es la norte, así como la noroeste y noreste, salvo Capula con exposición sureste y La Rosa con suroeste; estos últimos sitios se encuentran en cañadas protegidas, que propician condiciones adecuadas de humedad y sombra para la especie. Si se considera que las exposiciones sur son en general menos favorables, es importante conservar estos rodales, porque representan poblaciones casi únicas de la especie y pueden ser importantes en términos de adaptación o supervivencia en escenarios de cambio climático. Esto es particularmente relevante para la localidad de La Rosa que tiene un tamaño poblacional aceptable para su inclusión en un programa de conservación de la especie en la región.

Se encontraron seis diferentes unidades de suelo (Cuadro 3) (INEGI, 1988): regosol eútrico en 12 localidades (41\%); andosol ócrico, litosol, luvisol vértico y regosol dístrico en tres poblaciones cada uno; cambisol húmico y feozem háplico en dos localidades cada uno; y luvisol órtico en una. Los suelos resultaron de ácidos a ligeramente ácidos, con valores extremos de $\mathrm{pH}$ de 6.8 en Axopilco y Capula y de 4.8 en Cruz de Ocote. La materia orgánica osciló de 2.5 a $50.3 \%$, con valor promedio de $13 \%$. Las poblaciones estudiadas crecen en suelo de textura franco-arenosa (62\%), y el resto en suelo franco. Se encontró que $69 \%$ de los suelos en seco presentan un color pardo y sus combinaciones, donde el pardo grisáceo es el más común; los tonos gris y amarillo conforman el restante $31 \%$ con sus respectivas combinaciones. En suelo húmedo, el tono más común es el pardo grisáceo muy oscuro, y en menor proporción el gris con sus combinaciones. 
Se encontraron climas del tipo C (Cuadro 4), con predominio del $\mathrm{Cb}^{\prime}$ (w2), semifrío sub-húmedo con lluvias mayormente en verano y 5-10 \% de lluvia invernal del total anual, en 13 poblaciones (44.8\%). El clima C(w1) se encontró en nueve poblaciones, en los municipios de Mineral del Chico, Pachuca, Mineral del Monte y Singuilucan del Edo. de Hidalgo, y en Ixtacamaxtitlán y Altzayanca en el Edo. de Tlaxcala. El clima C(w2) se encontró en seis localidades; en Capula, Hgo. se encontró el $\mathrm{C}(\mathrm{m})$. Los valores de precipitación oscilan de 611 a $1109 \mathrm{~mm}$, con un valor promedio de $761 \mathrm{~mm}$ anuales. El valor mínimo se encontró en Vicente Guerrero, Hgo., en un bosque de encino, y el valor más alto en Plan del Baile, Pue., en zona de bosque de Abies religiosa (Kunth) Schltdl. et Cham. a una altitud de $3400 \mathrm{~m}$. Los valores de temperatura media anual varían de 10.1 a $14.0{ }^{\circ} \mathrm{C}$, con un valor promedio de $11.5^{\circ} \mathrm{C}$; el valor más bajo fue en Minatitlán, Pue., y el más alto en Vicente Guerrero, Hgo.

\begin{tabular}{|c|c|c|c|c|}
\hline Población & Municipio, Estado & Latitud $^{\dagger}$ & Longitud $^{\dagger}$ & Superficie (ha) $)^{\dagger \dagger}$ \\
\hline 1. Capula & Mineral del Chico, Hgo. & $20^{\circ} 12^{\prime} 45^{\prime \prime}$ & $98^{\circ} 47^{\prime} 15^{\prime \prime}$ & 15.0 \\
\hline 2. Las Antenas & Mineral del Chico, Hgo. & $20^{\circ} 11^{\prime} 39^{\prime \prime}$ & $98^{\circ} 44^{\prime} 19^{\prime \prime}$ & 4.0 \\
\hline 3. San José Capulines & Mineral del Chico, Hgo. & $20^{\circ} 10^{\prime} 50^{\prime \prime}$ & $98^{\circ} 47^{\prime} 49^{\prime \prime}$ & 32.4 \\
\hline 4. Estanzuela & Pachuca, Hgo. & $20^{\circ} 10^{\prime} 31^{\prime \prime}$ & $98^{\circ} 46^{\prime} 05^{\prime \prime}$ & 9.5 \\
\hline 5. Presa Jaramillo & Mineral del Chico, Hgo. & $20^{\circ} 10^{\prime} 18^{\prime \prime}$ & $98^{\circ} 44^{\prime} 00^{\prime \prime}$ & 22.4 \\
\hline 6. Vicente Guerrero & Omitlán de Juárez, Hgo. & $20^{\circ} 09^{\prime} 28^{\prime \prime}$ & $98^{\circ} 39^{\prime} 28^{\prime \prime}$ & 5.6 \\
\hline 7. Morán & Mineral del Monte, Hgo. & $20^{\circ} 08^{\prime} 50^{\prime \prime}$ & $98^{\circ} 39^{\prime} 40^{\prime \prime}$ & 0.5 \\
\hline 8. Peñas Cargadas & Mineral del Monte, Hgo. & $20^{\circ} 06^{\prime} 47^{\prime \prime}$ & $98^{\circ} 38^{\prime} 44^{\prime \prime}$ & 22.0 \\
\hline 9. Cuyamaloya & Singuilucan, Hgo. & $20^{\circ} 05^{\prime} 23^{\prime \prime}$ & $98^{\circ} 32^{\prime} 04^{\prime \prime}$ & 3.4 \\
\hline 10. El Salto & Singuilucan, Hgo. & $20^{\circ} 04^{\prime} 17^{\prime \prime}$ & $98^{\circ} 32^{\prime} 50^{\prime \prime}$ & 7.0 \\
\hline 11. Cañada El Atajo & Singuilucan, Hgo. & $20^{\circ} 03^{\prime} 20^{\prime \prime}$ & $98^{\circ} 32^{\prime} 53^{\prime \prime}$ & 5.4 \\
\hline 12. Tlaxco & Tlaxco, Tlax. & $19^{\circ} 39^{\prime} 00^{\prime \prime}$ & $98^{\circ} 03^{\prime} 11^{\prime \prime}$ & 48.5 \\
\hline 13. Villarreal & Terrenate, Tlax. & $19^{\circ} 33^{\prime} 24^{\prime \prime}$ & $97^{\circ} 54^{\prime} 30^{\prime \prime}$ & 78.0 \\
\hline 14. Zapata & Emiliano Zapata, Tlax. & $19^{\circ} 32^{\prime} 49^{\prime \prime}$ & $97^{\circ} 56^{\prime} 31^{\prime \prime}$ & 52.1 \\
\hline 15. La Rosa & Terrenate, Tlax. & $19^{\circ} 31^{\prime} 57^{\prime \prime}$ & $97^{\circ} 55^{\prime} 03^{\prime \prime}$ & 31.4 \\
\hline 16. San Juan & Terrenate, Tlax. & $19^{\circ} 30^{\prime} 58^{\prime \prime}$ & $97^{\circ} 55^{\prime} 16^{\prime \prime}$ & 12.3 \\
\hline 17. Axopilco & Altzayanca, Tlax. & $19^{\circ} 27^{\prime} 42^{\prime \prime}$ & $97^{\circ} 46^{\prime} 20^{\prime \prime}$ & 7.5 \\
\hline 18. Buenavista & Altzayanca, Tlax. & $19^{\circ} 25^{\prime} 02^{\prime \prime}$ & $97^{\circ} 50^{\prime} 04^{\prime \prime}$ & 45.0 \\
\hline 19. Cruz de Ocote & Ixtacamaxtitlán, Pue. & $19^{\circ} 42^{\prime} 24^{\prime \prime}$ & $97^{\circ} 51^{\prime} 47^{\prime \prime}$ & 18.4 \\
\hline 20. El Llanete & Ixtacamaxtitlán, Pue. & $19^{\circ} 34^{\prime} 51^{\prime \prime}$ & $97^{\circ} 52^{\prime} 33^{\prime \prime}$ & 28.6 \\
\hline 21. Minatitlán & Ixtacamaxtitlán, Pue. & $19^{\circ} 33^{\prime} 19^{\prime \prime}$ & $97^{\circ} 51^{\prime} 14^{\prime \prime}$ & 12.7 \\
\hline 22. Tlalmotolo & Ixtacamaxtitlán, Pue. & $19^{\circ} 32^{\prime} 59^{\prime \prime}$ & $97^{\circ} 43^{\prime} 51^{\prime \prime}$ & 40.5 \\
\hline 23. Tonalapa & Ixtacamaxtitlán, Pue. & $19^{\circ} 32^{\prime} 55^{\prime \prime}$ & $97^{\circ} 47^{\prime} 47^{\prime \prime}$ & 36.7 \\
\hline 24. Cuatexmola & Ixtacamaxtitlán, Pue. & $19^{\circ} 30^{\prime} 22^{\prime \prime}$ & $97^{\circ} 50^{\prime} 21^{\prime \prime}$ & 14.2 \\
\hline 25. La Caldera & Ixtacamaxtitlán, Pue. & $19^{\circ} 30^{\prime} 21^{\prime \prime}$ & $97^{\circ} 52^{\prime} 10^{\prime \prime}$ & 20.9 \\
\hline 26. La Garita & Ixtacamaxtitlán, Pue. & $19^{\circ} 29^{\prime} 10^{\prime \prime}$ & $97^{\circ} 49^{\prime} 00^{\prime \prime}$ & 3.6 \\
\hline 27. Apizaquito & Lafragua, Pue. & $19^{\circ} 10^{\prime} 12^{\prime \prime}$ & $97^{\circ} 20^{\prime} 41^{\prime \prime}$ & 18.5 \\
\hline 28. Barranca Canoita & Lafragua, Pue. & $19^{\circ} 10^{\prime} 57^{\prime \prime}$ & $97^{\circ} 19^{\prime} 07^{\prime \prime}$ & 13.3 \\
\hline 29. Plan del Baile & Tlachichuca, Pue. & $19^{\circ} 10^{\prime} 11^{\prime \prime}$ & $97^{\circ} 18^{\prime} 00^{\prime \prime}$ & 72.3 \\
\hline
\end{tabular}

'Dato en la parte central del rodal. ${ }^{\dagger}$ Estimadas mediante ArcMap 8x ${ }^{\circledR}$ en mapas de escala 1:50 000. 


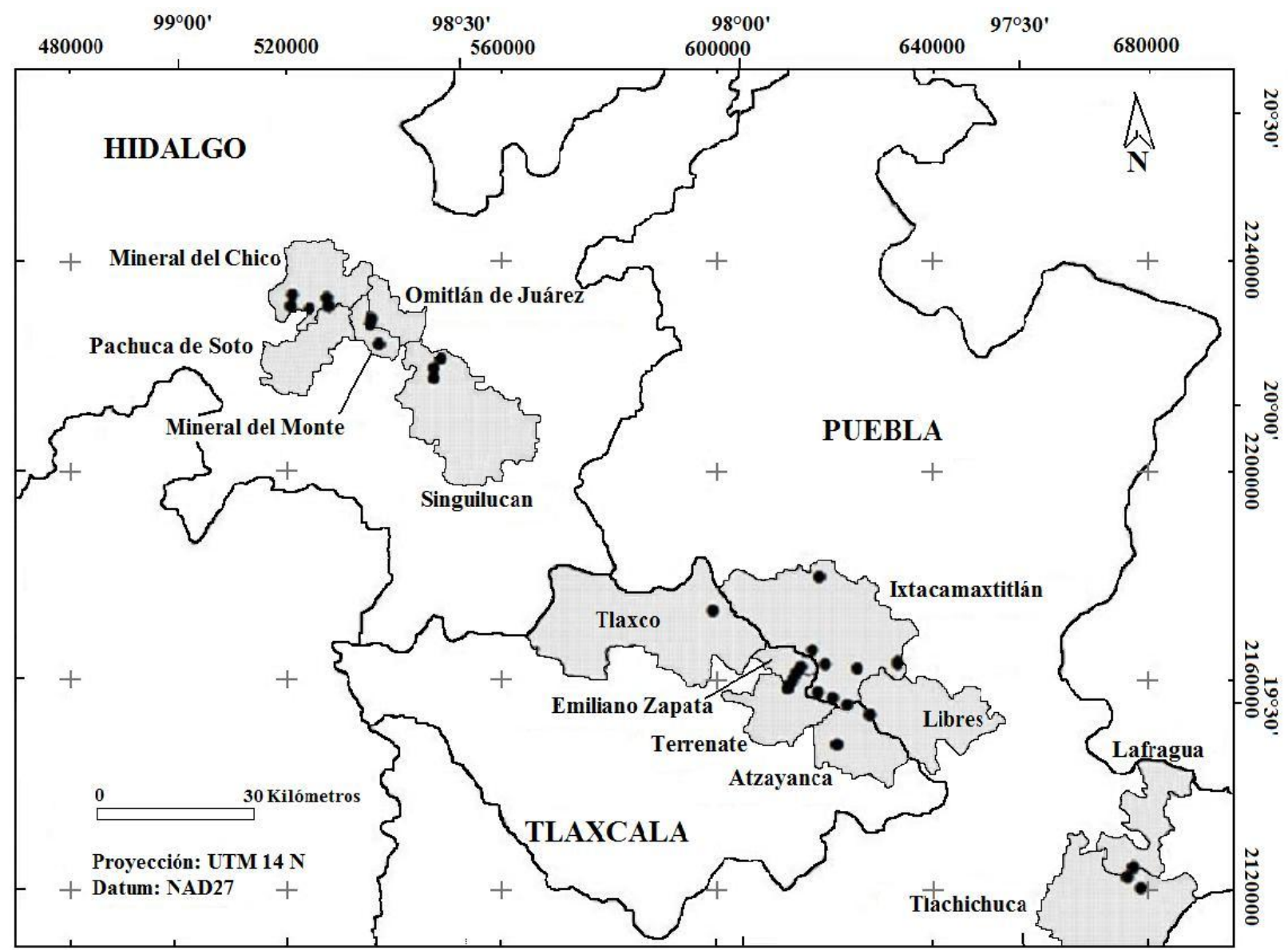

Figura 1. Ubicación geográfica de poblaciones de Pseudotsuga menziesii (Mirb.) Franco en los Estados de Hidalgo, Tlaxcala y Puebla, México.

Cuadro 2. Factores topográficos y fisiográficos donde se desarrollan las poblaciones naturales de Pseudotsuga menziesii (Mirb.) Franco en la zona centro de México.

\begin{tabular}{|c|c|c|c|c|c|c|c|}
\hline Población & Altitud (m) & Exposición & Pendiente $(\%)$ & Población & Altitud (m) & Exposición & Pendiente $(\%)$ \\
\hline 1. Capula & 2760 & $\mathrm{SE}$ & $60-75$ & 16. San Juan & 3100 & $\mathrm{NO}$ y $\mathrm{N}$ & $40-50$ \\
\hline 2. Las Antenas & 2900 & $\mathrm{O}$ & $70-80$ & 17. Axopilco & 2860 & NO & $80-90$ \\
\hline 3. San José Capulines & 2850 & $\mathrm{NE}$ & $60-75$ & 18. Buenavista & 2900 & $\mathrm{NO}$ y $\mathrm{N}$ & $40-55$ \\
\hline 4. Estanzuela & 2650 & $\mathrm{~N}$ y E & $65-75$ & 19. Cruz de Ocote & 2750 & $\mathrm{~N}$ & $50-70$ \\
\hline 5. Presa Jaramillo & 2850 & NO y NE & $65-75$ & 20. El Llanete & 3000 & $\mathrm{~N}, \mathrm{NO}$ & $60-70$ \\
\hline 6. Vicente Guerrero & 2450 & NO & $50-80$ & 21. Minatitlán & 2900 & $\mathrm{NE}$ & $45-50$ \\
\hline 7. Morán & 2600 & NO & $35-40$ & 22. Tlalmotolo & 2900 & NO y NE & $60-80$ \\
\hline 8. Peñas Cargadas & 2950 & NO & $20-60$ & 23. Tonalapa & 2750 & $\mathrm{~N}$ & $80-90$ \\
\hline 9. Cuyamaloya & 2650 & NO & $50-70$ & 24. Cuatexmola & 2850 & $\mathrm{~N}$ y $\mathrm{OE}$ & $40-50$ \\
\hline 10. El Salto & 2960 & NO & $55-70$ & 25. La Caldera & 3000 & $\mathrm{NO}$ & $45-55$ \\
\hline 11. Cañada El Atajo & 2650 & $\mathrm{~N}$ & $50-70$ & 26. La Garita & 2980 & $\mathrm{NE}$ & $50-60$ \\
\hline 12. Tlaxco & 3050 & $\mathrm{~N}$ & $50-80$ & 27. Apizaquito & 3200 & $\mathrm{NO}$ & $50-80$ \\
\hline 13. Villarreal & 2950 & $\mathrm{~N}$ y NE & $60-65$ & 28. Barranca Canoita & 3250 & NO & $60-75$ \\
\hline 14. Zapata & 2990 & $\mathrm{NO}$ y N & $70-80$ & 29. Plan del Baile & 3400 & NO & $45-80$ \\
\hline 15. La Rosa & 2950 & SO & $60-75$ & & & & \\
\hline
\end{tabular}


Cuadro 3. Factores edáficos donde se desarrollan las poblaciones naturales de Pseudotsuga menziesii (Mirb.) Franco en la zona centro de México.

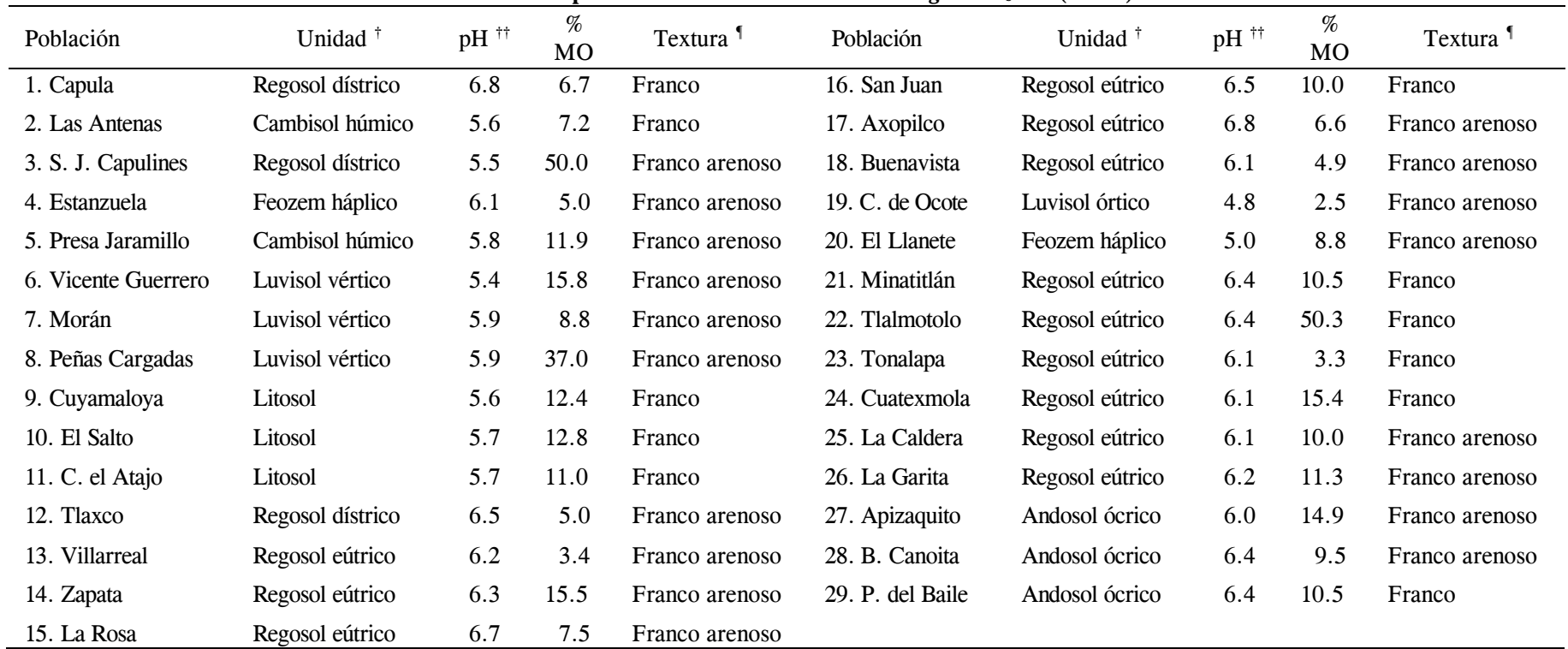

†INEGI, 1988; ${ }^{\dagger \dagger}$ Solución acuosa (2:1); ${ }^{\uparrow}$ Método de Bouyucos.

Cuadro 4. Tipo de clima, precipitación media anual, temperatura media anual e índice de aridez de las poblaciones de Pseudotsuga menziesii (Mirb.) Franco en la zona centro de México.

\begin{tabular}{|c|c|c|c|c|c|c|c|c|c|}
\hline Población & Clima $^{\dagger}$ & $\begin{array}{l}\mathrm{PP}^{\dagger \dagger} \\
(\mathrm{mm})\end{array}$ & $\begin{array}{c}\mathrm{T}^{\dagger \dagger} \\
\left({ }^{\circ} \mathrm{C}\right)\end{array}$ & $\begin{array}{l}\text { Índice } \\
\text { aridez }\end{array}$ & Población & $\mathrm{Clima}^{\dagger}$ & $\begin{array}{l}\mathrm{PP}^{\dagger \dagger} \\
(\mathrm{mm})\end{array}$ & $\begin{array}{c}\mathrm{T}^{\dagger \dagger} \\
\left({ }^{\circ} \mathrm{C}\right) \\
\end{array}$ & $\begin{array}{l}\text { Índice } \\
\text { aridez }\end{array}$ \\
\hline 1. Capula & $\mathrm{C}(\mathrm{m})$ & 668 & 13.0 & 4.2 & 16. San Juan & $\mathrm{Cb}^{\prime}(\mathrm{w} 2)$ & 766 & 11.3 & 3.0 \\
\hline 2. Las Antenas & $\mathrm{C}(\mathrm{w} 2)$ & 763 & 11.9 & 3.3 & 17. Axopilco & $\mathrm{C}(\mathrm{w} 1)$ & 837 & 10.8 & 2.6 \\
\hline 3. S. J. Capulines & $\mathrm{C}(\mathrm{w} 1)$ & 743 & 12.2 & 3.6 & 18. Buenavista & $\mathrm{C}(\mathrm{w} 1)$ & 707 & 11.3 & 3.3 \\
\hline 4. Estanzuela & $\mathrm{C}(\mathrm{w} 1)$ & 638 & 13.6 & 4.9 & 19. C. de Ocote & $\mathrm{C}(\mathrm{w} 1)$ & 665 & 12.2 & 4.0 \\
\hline 5. Presa Jaramillo & $\mathrm{C}(\mathrm{w} 2)$ & 715 & 12.4 & 3.8 & 20. El Llanete & $\mathrm{Cb}^{\prime}(\mathrm{w} 2)$ & 811 & 11.1 & 2.8 \\
\hline 6. Vicente Guerrero & $\mathrm{C}(\mathrm{w} 2)$ & 611 & 14.0 & 6.0 & 21. Minatitlán & $\mathrm{Cb}^{\prime}(\mathrm{w} 2)$ & 896 & 10.1 & 2.1 \\
\hline 7. Morán & $\mathrm{C}(\mathrm{w} 2)$ & 617 & 13.5 & 5.0 & 22. Tlalmotolo & $\mathrm{Cb}^{\prime}(\mathrm{w} 2)$ & 726 & 11.7 & 3.4 \\
\hline 8. Peñas Cargadas & $\mathrm{C}(\mathrm{w} 1)$ & 740 & 11.8 & 3.4 & 23. Tonalapa & $\mathrm{Cb}^{\prime}(\mathrm{w} 2)$ & 824 & 10.8 & 2.6 \\
\hline 9. Cuyamaloya & $\mathrm{C}(\mathrm{w} 1)$ & 635 & 13.1 & 4.7 & 24. Cuatexmola & $\mathrm{Cb}^{\prime}(\mathrm{w} 2)$ & 738 & 11.5 & 3.6 \\
\hline 10. El Salto & $\mathrm{C}(\mathrm{w} 1)$ & 732 & 11.7 & 3.4 & 25. La Caldera & $\mathrm{Cb}^{\prime}(\mathrm{w} 2)$ & 683 & 11.7 & 3.3 \\
\hline 11. C. El Atajo & $\mathrm{C}(\mathrm{w} 1)$ & 617 & 13.1 & 4.8 & 26. La Garita & $\mathrm{Cb}^{\prime}(\mathrm{w} 2)$ & 791 & 10.9 & 2.8 \\
\hline 12. Tlaxco & $\mathrm{C}(\mathrm{w} 2)$ & 729 & 12.1 & 3.5 & 27. Apizaquito & $\mathrm{Cb}^{\prime}(\mathrm{w} 2)$ & 869 & 10.3 & 2.3 \\
\hline 13. Villarreal & $\mathrm{Cb}^{\prime}(\mathrm{w} 2)$ & 857 & 10.6 & 2.4 & 28. B. Canoita & $\mathrm{Cb}^{\prime}(\mathrm{w} 2)$ & 945 & 9.9 & 2.0 \\
\hline 14. Zapata & $\mathrm{Cb}^{\prime}(\mathrm{w} 2)$ & 837 & 10.7 & 2.5 & 29. Plan del Baile & $\mathrm{Cb}^{\prime}(\mathrm{w} 2)$ & 1109 & 8.9 & 1.4 \\
\hline 15. La Rosa & $\mathrm{C}(\mathrm{w} 2)$ & 811 & 11.1 & 2.8 & & & & & \\
\hline
\end{tabular}

${ }^{\dagger}$ Clasificación climática de Köppen modificada por García (1989).

${ }^{\dagger} \mathrm{PP}=$ precipitación y $\mathrm{T}=$ temperatura media anual, http://forest.moscowfsl.wsu.edu/climate.

Los tipos de vegetación donde se desarrolla $P$. menziesii son el bosque de oyamel, bosque de pino, bosque de pinoencino y bosque de encino-pino; el más frecuente es el de oyamel y de pino con 11 y 10 localidades, respectivamente (Cuadro 5). En cuatro poblaciones domina el bosque de encino, en tres el de pino-encino, y en una el de encino-pino. Así, la especie arbórea asociada con mayor frecuencia (25 poblaciones) a Pseudotsuga es Abies religiosa. También se asocian varias especies de los géneros Quercus y Pinus, y en menor grado Arbutus y Juniperus. Además, $P$. menziesii presenta escasa repobla- ción natural en comparación con otras especies que ahí habitan, como Quercus y Abies. La población que presenta mayor número de individuos adultos de Pseudotsuga es San José Capulines, con 1450 árboles, mientras que la más pequeña es Moran con sólo 4 árboles, ambas en el Estado de Hidalgo. Se estimó un total de 9029 árboles adultos (311 en promedio), 14 poblaciones con menos de 200 árboles, y de estás, seis con menos de 50 individuos. 
Cuadro 5. Tipos de vegetación y especies arbóreas asociadas con las poblaciones de Pseudotsuga menziesii (Mirb.) Franco en la zona centro de México.

\begin{tabular}{|c|c|c|c|}
\hline Población & $\begin{array}{c}\text { Tipo de vegetación. } \\
\text { Bosque de: }\end{array}$ & $\begin{array}{c}\text { Especies arbóreas } \\
\text { asociadas }^{\dagger}\end{array}$ & $\begin{array}{c}\mathrm{N}^{\circ} \text { total de árboles } \\
\text { adultos de Pseudotsuga }\end{array}$ \\
\hline 1. Capula & Encino v/s & Qa, Ql, Qr, Ar, Lg & 460 \\
\hline 2. Las Antenas & Oyamel & Ar, Qsp & 25 \\
\hline 3. San José Capulines & Encino v/s & Qr, Q1, Jd, Ax, Ar & 1450 \\
\hline 4. Estanzuela & Oyamel & Ar, Qsp, Jd, Ax & 460 \\
\hline 5. Presa Jaramillo & Oyamel & Ar, Asp, Jm, Pt, Qr, Qm & 450 \\
\hline 6. Vicente Guerrero & Encino & Qsp, Ar, Jd & 16 \\
\hline 7. Morán & Encino & Ql, Qr, Ar, Ax, Cm, Jd, Pt, Qc & 4 \\
\hline 8. Peñas Cargadas & Oyamel & Ar, Qc, Qsp, Pt, Jd & 525 \\
\hline 9. Cuyamaloya & Pino & Ppa, Qr, Asp & 52 \\
\hline 10. El Salto & Encino-pino & Pp, Pr, Qsp & 105 \\
\hline 11. Cañada El Atajo & Pino-encino & $\mathrm{Pt}, \mathrm{Ql}, \mathrm{Qm}$ & 35 \\
\hline 12.Tlaxco & Pino & $\mathrm{Pr}, \mathrm{Pt}, \mathrm{Pa}, \mathrm{Ar}, \mathrm{Ax}, \mathrm{Qsp}$ & 400 \\
\hline 13. Villarreal & Oyamel & Ar, Ql, Qr, Qsp, Pt, Pp, Pa, Ax, Aj & 700 \\
\hline 14. Zapata & Oyamel & $\mathrm{Ar}, \mathrm{Ppa}, \mathrm{Pp}, \mathrm{Pt}, \mathrm{Pa}$ & 612 \\
\hline 15. La Rosa & Pino & Pt, Pr, Pm, Pp, Pa, Ar & 165 \\
\hline 16. San Juan & Pino & $\mathrm{Pp}, \mathrm{Pa}, \mathrm{Qr}, \mathrm{Ax}, \mathrm{Ar}$ & 84 \\
\hline 17. Axopilco & Pino-encino & $\mathrm{Pp}, \mathrm{Pa}, \mathrm{Ar}, \mathrm{Qsp}$ & 370 \\
\hline 18. Buenavista & Pino-encino & $\mathrm{Cl}, \mathrm{Po}, \mathrm{Ar}, \mathrm{Qsp}, \mathrm{Pt}, \mathrm{Ax}$ & 60 \\
\hline 19. Cruz de Ocote & Pino & $\mathrm{Pt}, \mathrm{Pp}, \mathrm{Ppa}, \mathrm{Pa}, \mathrm{Qr}$ & 85 \\
\hline 20. El Llanete & Pino v/s & Pt, Ql, Qr, Ar & 450 \\
\hline 21. Minatitlán & Oyamel & $\mathrm{Pa}, \mathrm{Ppa}, \mathrm{Ar}, \mathrm{Qr}, \mathrm{Ql}$, Qcra & 45 \\
\hline 22. Tlalmotolo & Pino & $\mathrm{Pa}, \mathrm{Ppa}, \mathrm{Pp}, \mathrm{Ar}, \mathrm{Qr}$ & 565 \\
\hline 23. Tonalapa & Oyamel & $\mathrm{Pp}, \mathrm{Ppa}, \mathrm{Pa}, \mathrm{Qr}, \mathrm{Ql}, \mathrm{Ar}$ & 166 \\
\hline 24. Cuatexmola & Pino & $\mathrm{Pt}, \mathrm{Pa}, \mathrm{Pp}, \mathrm{Ar}, \mathrm{Qsp}$ & 250 \\
\hline 25. La Caldera & Pino & $\mathrm{Pt}, \mathrm{Pp}, \mathrm{Ar}$ & 380 \\
\hline 26. La Garita & Oyamel & Ar, Pp, Qsp & 15 \\
\hline 27. Apizaquito & Pino & $\mathrm{Pp}, \mathrm{Pa}, \mathrm{Ar}, \mathrm{Qsp}$ & 210 \\
\hline 28. Barranca Canoita & Oyamel & Ar, Qr, Ql, Ax, Aj, Pp & 65 \\
\hline 29. Plan del Baile & Oyamel & Ar, Pm, Pt, Pp, Po, Ql, Ax, Jm, Af & 825 \\
\hline
\end{tabular}

v/s, con vegetación secundaria; ${ }^{\dagger} \mathrm{Af}$ (Alnus firmifolia); $\mathrm{Aj}$ (Alnus jorulensis); $\mathrm{Ar}$ (Abies religiosa); Asp (Arbutus sp); Ax (Arbutus xalapensis); $\mathrm{Cl}$ (Cupresus lusitanica); Cm (Crataegus mexicana); Jd (Juniperus deppeana); Jm (Juniperus monticola); Lg (Litsea glaucescens); Pa (Pinus ayacahuite); Pm (Pinus montezumae); Po (Pinus oaxacana); Pp (Pinus pseudostrobus); Ppa (Pinus patula); Pr (Pinus rudis); Pt (Pinus teocote); Q1 (Quercus laurina); Qa (Quercus affinis); Qc (Quercus crassipes); Qcra (Quercus crassifolia); Qm (Quercus mexicana); Qr (Quercus rugosa); Qsp (Quercus sp).

\section{Agrupamiento de las poblaciones}

Se generó el dendrograma de asociación de poblaciones (Figura 2) con base en siete variables eco-geográficas: latitud, longitud, tipo de clima, especie arbórea asociada, índice de aridez, pH y textura del suelo. La máxima distancia euclidiana entre grupos resultó de 1.175 . La separación de poblaciones permite dilucidar dos grandes zonas de Pseudotsuga mensiezii en el centro de México. La Zona I con 11 poblaciones (de la 1 a la 11) y la Zona II, con las 18 poblaciones restantes. $\mathrm{Al}$ hacer un corte a $50 \%$ de esa distancia, a un valor de 0.587 (línea punteada en Figura 2), es posible establecer la formación de seis grupos en cada zona (Cuadro 6). La Zona I comprende las 11 poblaciones que se desarrollan en el Estado de
Hidalgo, con clima predominante $\mathrm{C}(\mathrm{w} 1)$ o $\mathrm{C}(\mathrm{w} 2)$, con una elevación de 2450 a 2960 m y un promedio de 2750 $\mathrm{m}$. La temperatura media anual de esta zona varía de 11.7 a $15.0{ }^{\circ} \mathrm{C}$, con una media de $12.8{ }^{\circ} \mathrm{C}$. La precipitación media anual de esta zona varía de 611 a $763 \mathrm{~mm}$, con un promedio de $680 \mathrm{~mm}$. El índice de aridez varía de 3.3 a 6, con un promedio de 4.3. Los suelos están dominados por litosoles, luvisoles, cambisoles, regosoles y feozems, con textura franco-arenosa a franco, y un $\mathrm{pH}$ que oscila de 5.4 a 6.8 , con un valor promedio de 5.8. Las especies asociadas más comunes son Abies religiosa y algunas especies del género Quercus. 


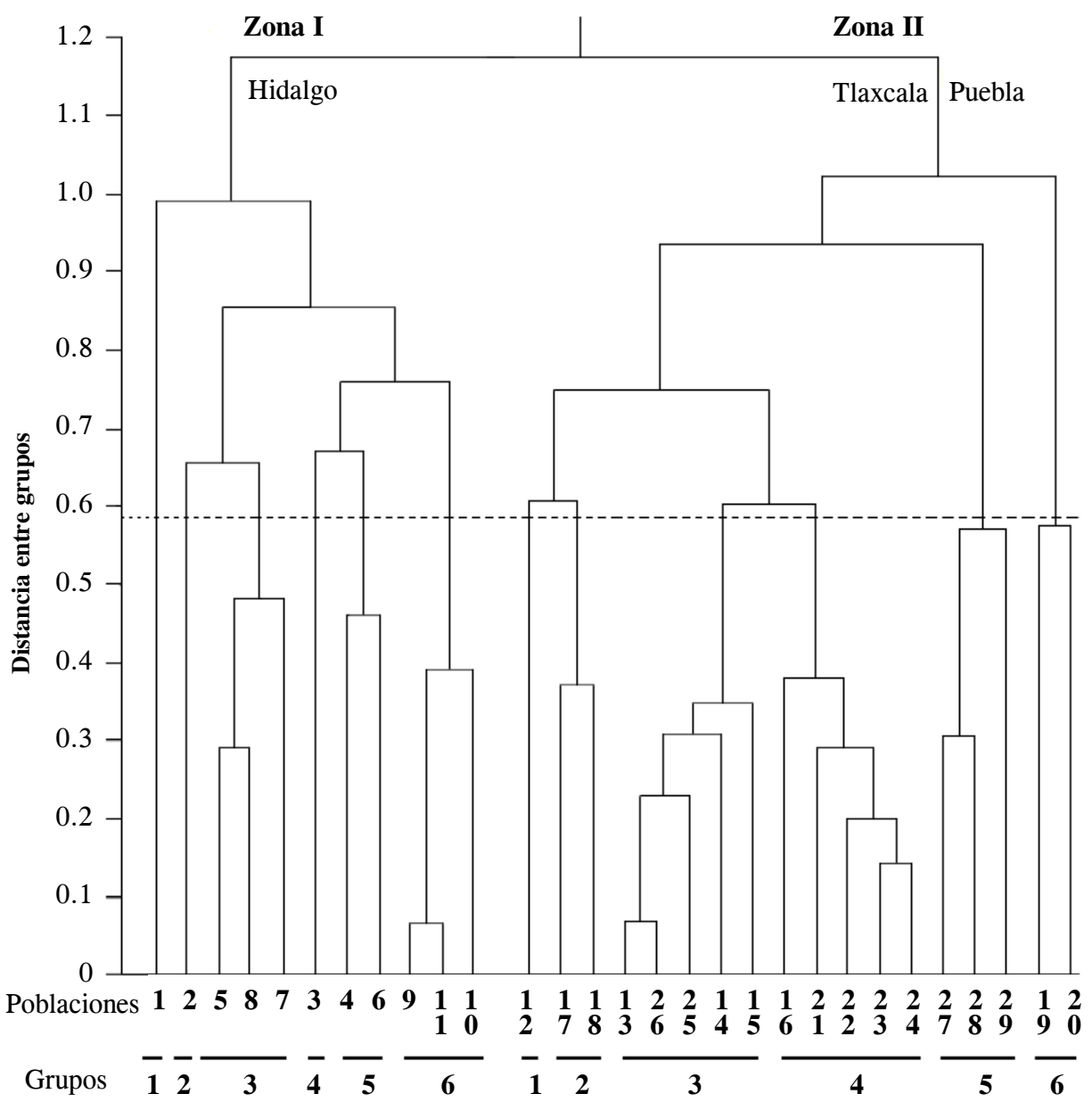

Figura 2. Dendrograma de las 29 poblaciones de Pseudotsuga menziesii (Mirb.) Franco de la zona centro de México, basado en un análisis de conglomerados de siete variables biofísicas. Los nombres de las poblaciones se indican en Cuadro 1.

Cuadro 6. Grupos de poblaciones en dos zonas del centro de México, donde se ubica Pseudotsuga menziesii (Mirb.) Franco según el análisis de agrupamiento.

\begin{tabular}{ll}
\hline Grupo & \multicolumn{1}{c}{ Población } \\
\hline & \multicolumn{1}{c}{ ZONA I } \\
I. 1 & Capula \\
I. 2 & Las Antenas \\
I. 3 & Presa Jaramillo, Peñas Cargadas, Morán \\
I. 4 & San José Capulines \\
I. 5 & Estanzuela, Vicente Guerrero \\
I. 6 & Cuyamaloya, El Salto, Cañada El Atajo \\
& \multicolumn{2}{c}{ ZONA II } \\
II. 1 & Tlaxco $\quad$ Axopilco, Buenavista \\
II. 2 & Villarreal, Zapata, La Rosa, La Caldera, La Garita \\
II. 3 & San Juan, Minatitlán, Tlalmotolo, Tonalapa, Cuatexmola \\
II. 4 & Apizaquito, Barranca Canoita, Plan del Baile \\
II. 5 & Cruz de Ocote, El Llanete \\
II. 6 & \\
\hline
\end{tabular}

La Zona II comprende las 18 poblaciones de los Estados de Tlaxcala y Puebla, con una elevación de 2750 a $3400 \mathrm{~m}$ y con un promedio de $2990 \mathrm{~m}$. La mayoría están en el clima $\mathrm{Cb}^{\prime}(\mathrm{w} 2)$. La temperatura media anual de esta zona es de 8.9 a $12.2{ }^{\circ} \mathrm{C}$, con una media de $11{ }^{\circ} \mathrm{C}$. La precipitación media anual de esta zona varía de 665 a $1109 \mathrm{~mm}$, con un promedio de $811 \mathrm{~mm}$. El índice de aridez varía de 1.4 a 4 , con promedio de 2.8. Los suelos están dominados por regosoles y andosoles, con dos casos de luvisol o feozem, de textura franco-arenosa a franco, y un $\mathrm{pH}$ que oscila de 4.8 a 6.8 con un valor promedio de 6.4. Las especie asociadas más comunes son Abies religiosa y varias del género Pinus.

\section{DISCUSIÓN}

Los datos de la distribución natural de la especie indican una alta fragmentación de las poblaciones en la región central de México, en una estructura similar a metapoblaciones (Mosseler, 1998), lo cual señala un alto nivel de aislamiento para la especie y sugiere un limitado 
intercambio genético entre estas poblaciones. La superficie promedio por población ( 25 ha) es baja, si se compara con las grandes extensiones de otras especies arbóreas de la región. El tamaño de las poblaciones varía de 4 a 1450 árboles adultos; 11 de esas poblaciones tienen menos de 100 árboles sexualmente maduros, con una media de 311 y una mediana de 210 árboles. Se ha estimado que se requiere un tamaño de población de al menos 180 árboles para reducir los efectos negativos de la falta de polinización y de endogamia para mantener la producción de semilla a niveles suficientes en especies de coníferas (O'Connell et al., 2006). En algunas de estas poblaciones se han determinado niveles altos de endogamia (Cruz et al., 2008), lo que concuerda con la escasa producción de semillas (Mápula et al., 2007), problemas de germinación y crecimiento inicial (Juárez et al., 2006; Mápula et al., 2008), así como una baja repoblación natural (Velasco et al., 2007), lo que fue comprobado visualmente en casi todas las poblaciones incluidas en este trabajo.

Una forma de recuperar la variabilidad genética para contrarrestar los efectos de la endogamia en la capacidad reproductiva (Mosseler, 1998; Mosseler et al., 2004) es fomentar el flujo genético entre poblaciones vecinas que se desarrollen en ambientes similares. El intercambio de genes con riesgos reducidos, por medio de la migración asistida, al hacer plantaciones de árboles con mayor posibilidad de adaptación en poblaciones vecinas, generaría un aumento de la diversidad genética (Ledig et al., 2000), así como mayor posibilidad de adaptación a cambios climáticos u otro factores adversos. Movimientos de material genético entre poblaciones cercanas reducen problemas de adaptación (depresión exogámica) y contrarrestan los efectos del aislamiento geográfico, al simular lo que ocurriría de manera natural si no existiera la fragmentación actual.

Debido a las diferentes condiciones ambientales entre las dos zonas de poblaciones, no sería recomendable transferir semilla o plantas entre ellas. Se sugiere llevar a cabo migración asistida sólo dentro de cada grupo de poblaciones (estrategia conservadora), para propiciar un movimiento más cercano a lo natural y simular el flujo genético. Sin embargo, las poblaciones de Capula, Las Antenas, San José Capulines y Tlaxco resultan únicas, porque por sí mismas representan un grupo, y por tanto tiene características exclusivas; en este caso la transferencia de semilla sería riesgosa, especialmente para Capula que resultó con la mayor distancia euclidiana para ligarse a las demás poblaciones en su zona, la de Hidalgo. Estos análisis de agrupamiento ayudan también a definir condiciones ambientales donde la especie puede prosperar adecuadamente, como una estrategia de conservación ex situ (dinámica), a través de ensayos o plantaciones de conservación. También sirve para proponer lugares adecuados para el establecimiento de plantaciones de árboles de Navidad.

La zonificación y los grupos de poblaciones propuestos definitivos, así como las procedencias adecuadas para las plantaciones ex situ deberían establecerse hasta que se hayan realizado ensayos de procedencias, en los que todas estas poblaciones sean evaluadas en ambiente común para determinar su nivel de adaptación y crecimiento. Dado el reducido número de individuos por población se requiere establecer un programa de conservación in situ de la especie y su hábitat para conservación de sus genes y su potencial uso de manera comercial.

La población de Morán es de interés particular por ser la población tipo que señala Flous (1934) para describir a $P$. macrolepis Flous. A pesar de que se ha establecido que solo hay una especie en México de Pseudotsuga, que es P. menziesii (Reyes et al., 2005), es importante mantener y proteger cualquier localidad que representa parte del acervo genético de esta conífera, especialmente cuando estas poblaciones podrían representar variedades de la especie (Debreczy y Rácz, 1995; Reyes et al., 2006).

\section{CONCLUSIONES}

En la zona central de México se ubican 29 poblaciones de Pseudotsuga menziesii en los estados de Hidalgo, Tlaxcala y Puebla, con un estimado de 9029 árboles adultos en 682 ha, en lugares con exposición preferente al norte, en cañadas relativamente protegidas y de pendiente fuerte. Estas poblaciones se ubican entre los 2450 y 3400 msnm, especialmente en bosques donde dominan Abies religiosa o varias especies de Pinus en las localidades más frías, o especies de Quercus, particularmente en Hidalgo, donde hay mayor índice de aridez. Por su similitud ambiental y cercanía geográfica las poblaciones se agrupan en dos grandes regiones, 11 en el Estado de Hidalgo (Zona I) y 18 en los Estados de Tlaxcala y Puebla (Zona II). Dentro de cada zona se distinguen seis grupos con mayor afinidad ambiental; sin embargo, cuatro grupos se integran con una sola población porque reflejan condiciones ambientales distintas. Podrían existir riesgos de adaptación si se introduce material de poblaciones de diferentes grupos.

\section{AGRADECIMIENTOS}

Al proyecto de investigación "Conservación y mejoramiento genético de Pseudotsuga spp.; Conífera estratégica del estado de Tlaxcala y la región central del 
país" (clave 2002-C01-6416) que se efectuó con apoyo económico del Fondo Sectorial CONAFOR-CONACYT.

\section{BIBLIOGRAFÍA}

Acevedo R R, J J Vargas H, J López U, J Velázquez M (2006) Efecto de la procedencia geográfica y de la fertilización en la fenología del brote terminal en plántulas de Pseudotsuga sp. Agrociencia 40:125-137.

Álvarez M J G, I Alia T, M T Colinas L, J Sahagún C (2007) Interspecific differences in postharvest quality on Mexican Christmas trees. Silva Genet. 56:65-73.

Comisión Nacional Forestal, CONAFOR (2007) Importación de árboles de navidad. Revista México Forestal, No. 53. Disponible en: http://www.mexicoforestal.gob.mx/index.php (Octubre 2007).

Cruz N J, J J Vargas H, P Ramírez V, J López U (2008) Patrón de cruzamiento en poblaciones naturales de Pseudotsuga menziesii (Mirb.) Franco, en México. Agrociencia 42:367-378.

Debreczy Z, I Rácz (1995) New species and varieties of conifers from Mexico. Phytologia 78:217-243.

Del Castillo R F, J A Pérez de la R, G Vargas A, R Rivera G (2004) Coníferas. In: Biodiversidad de Oaxaca. A J García M, M de J Ordóñez, M Briones S (eds). Instituto de Biología, UNAMFondo Oaxaqueño para la Conservación de la NaturalezaWorld Wildlife Foundation. pp:141-158.

Domínguez A F A (1994) Análisis Histórico-Ecológico de los Bosques de Pseudotsuga en México. INIFAP-CIR Golfo Centro. Folleto Técnico No 23. El Palmar, Veracruz. 43 p.

Domínguez A F A, J J Vargas H, J López U, P Ramírez V, E Guízar N (2004) Caracterización ecológica de Pseudotsuga menziesii en Pinal de Amoles, Querétaro: nueva población natural en México. Anal. Inst. Biol. (UNAM) 75:191-203.

Flous F (1934) Deux espèces nouvelles de Pseudotsuga Américains. Bull. Soc. Hist. Nat. Toulouse 66:211-224.

García E (1989) Apuntes de Climatología. 6ta ed. Offset Larios, México, DF. 155 p.

Hermann R K, D P Lavender (1999) Douglas-fir planted forests. New For. 17:53-70.

Hutchinson M F (2004) ANUSPLINE Version 4.3 User Guide. Centre for Resource and Environmental Studies. The Australian National University, Canberra. $54 \mathrm{p}$.

Juárez A A, J López U, J J Vargas H, C Sáenz R (2006) Variación geográfica en la germinación y crecimiento inicial de plántulas de Pseudotsuga menziesii de México. Agrociencia 40:783-792.

Instituto Nacional de Geografía y Estadística, INEGI (1988) Atlas del Medio Físico. Instituto Nacional de Estadística, Geografía e Informática. Aguascalientes, México. pp:98-99, 110-111, $128-126$

Ledig F T, M Mápula L, B Bermejo V, J V Reyes H, C Flores L, M A Capó A (2000) Locations of endangered spruce populations in Mexico and the demography of Picea chihuahuana. Madroño 47:71-88.

Mápula L M, J López U, J J Vargas H, A Hernández L (2007) Reproductive indicators in natural populations of Douglas-fir in Mexico. Biodiv. Conserv. 16:727-742.
Mápula L M, J López U, J J Vargas H, A Hernández L (2008) Germinación y vigor de semillas de Pseudotsuga menziesii de México. Ra Ximhai 4:119-134.

Mosseler A (1998) Minimum viable population size and the conservation of forest genetics resources. In: Tree Improvement: Applied Research and Technology Transfer. S Puri (ed). Science Publishers Inc. Enfield, NH, USA. pp:191205.

Mosseler A, O P Rajora, J E Major, K H Kim (2004) Reproductive and genetic characteristics of rare, disjunct pitch pine populations at the northern limits of its range in Canada. Conserv. Genet. 5:571-583.

Munsell A H (1990) Soil Color Charts. MacBeth Division of Kollmorgen Instruments Corp. Baltimore, Maryland, USA.

O'Conell L M, A Mosseler, O P Rajora (2006) Impacts of forest fragmentation on the reproductive success of white spruce (Picea glauca). Can. J. Bot. 84:956-965.

Rehfeldt G E (2006) A Spline Climate Model for Western United States. General Technical Report 165, USDA, Forest Service, Rocky Mountain Research Station. Fort Collins, CO, USA. 21 p.

Reyes H V, J J Vargas H, J López U, H Vaquera H (2005) Variación morfológica y anatómica en poblaciones mexicanas de Pseudotsuga (Pinaceae). Acta Bot. Mex. 70:47-67.

Reyes H V, J J Vargas H, J López U, H Vaquera H (2006) Similitud fenotípica de poblaciones mexicanas de Pseudotsuga Carr. Agrociencia 40:545-556.

Sáenz R C, R R Guzmán R, G E Rehfeldt (2006) Altitudinal genetic among Pinus oocarpa populations in Michoacán, Mexico: Implications for seed zoning conservation, tree breeding and global warming. For. Ecol. Manage. 229:340-350.

Sanhueza A, M Bourke, H Grosse, I Chacón, P Álvarez (1998) Cultivo del Pino Oregón. Programa de Diversificación Forestal. Corporación Nacional Forestal. Chile. 106 p.

SAS Institute (1998) SAS User Guide. SAS Institute Inc. Cary, NC, USA. $595 \mathrm{p}$.

Secretaría de Medio Ambiente y Recursos Naturales, SEMARNAT (2002a) Norma Oficial Mexicana NOM-059-ECOL-2001. Diario Oficial, 2da Sección. Protección ambiental -Especies nativas de México de flora y fauna silvestres -Categorías de riesgo y especificaciones para su inclusión, exclusión o cambio -Lista de especie en riesgo. Marzo de 2002. México, D.F. 85 p.

Secretaría de Medio Ambiente y Recursos Naturales, SEMARNAT (2002b) Norma Oficial Mexicana NOM-021-RECNAT-2000. Diario Oficial, 2da Sección, que establece las especificaciones de fertilidad, salinidad y clasificación de suelos, estudio, muestreo y análisis. Diciembre de 2002. México, D.F. 85 p.

Sneath P H A, R R Sokal (1973) Numerical Taxonomy: The Principles and Practice of Numerical Classification. Freeman. San Francisco. 573 p.

van Reeuwijk L P (1999) Procedimientos para Análisis de Suelos. M C Gutiérrez C, C A Tavarez E, C A Ortiz S (trads). Especialidad de Edafología. Colegio de Postgraduados. Montecillo, México. $145 \mathrm{p}$

Velasco G M V, J López U, G Angeles P, J J Vargas H, V Guerra de la C (2007) Dispersión de semillas de Pseudotsuga menziesii en poblaciones del centro de México. Agrociencia 41:121-131. 